\title{
Effect of the route and sintering time in the microstructure of pure aluminum prepared by high energy ball milling
}

José Mendoza ${ }^{1}$, C. Carreño-Gallardo ${ }^{2}$, I. Estrada-Guel ${ }^{3}$, C.G. Garay-Reyes ${ }^{3}$, M.A. Ruiz-Esparza-Rodriguez ${ }^{3}$, G. Rodríguez-Cabriales ${ }^{3}$, J.C Guía-Tello ${ }^{3}$ and R. Martínez-Sánchez ${ }^{4}$

${ }^{1}$ Centro de InvestigaciónenMaterialesAvanzados, Chihuahua, Chihuahua, Mexico, ${ }^{2}$ Centro de InvestigaciónenMaterialesAvanzados (CIMAV), Chihuahua, Chihuahua, Mexico, ${ }^{3}$ Centro de InvestigaciónenMaterialesAvanzados (CIMAV), Laboratorio Nacional de Nanotecnología, Miguel de Cervantes No. 120, CP 31136, Chihuahua, Chih., México., Chihuahua, Chihuahua, Mexico, ${ }^{4}$ Centro de InvestigaciónenMaterialesAvanzados (CIMAV) Laboratorio Nacional de Nanotecnología, Miguel de Cervantes No. 120, CP 31136, Chihuahua, Chih., México., Chihuahua, Chihuahua, Mexico

Aluminum ( $\mathrm{Al}$ ) is a metal universally used with appreciated characteristics such as characteristics as low density and high corrosion resistance; however in pure form is not used as a structural material because of its modest mechanical performance. There are some routes to improve its mechanical response: grain size reduction, strain hardening, solid solution alloying and annealing. Mechanical milling (MM) induces severe plastic deformation (SPD) in some metallic systems, resulting in microstructure refinement [1]. Using SPD it is possible to obtain nanocrystalline (NC, solid with grain size $<100 \mathrm{~nm}$ ) or ultrafine-grained (UFG, materials with a grain size ranged from 100nm to $1000 \mathrm{~nm}$ ) samples [2]. However, this characteristic is negatively affected during sintering process because of the high temperature and prolonged holding time. High frequency induction sintering (HFIS) involves a short sintering time (minutes) avoiding an excessive growth in samples [3].

This work deals with the microstructural analysis of pure Al powders prepared by MM and sintered at different periods of time by HFIS. These samples were compared with a sample prepared using the conventional method (CM). Pure Al powders were milled for two hours in a SPEX 8000M mill using five drops of methanol as a process control agent to avoid the formation of agglomerates.

During the HFIS, the milled powders were compacted (450 MPa) and heated (at $450{ }^{\circ} \mathrm{C}$ ) simultaneously for 1 , $3,5,7$, and 10 minutes. On the other hand, using CM, the powders were cold compacted (900 MPa) for 3 minutes and subsequently were sintered in a conventional furnace at $550{ }^{\circ} \mathrm{C}$ for 3 hours. The sintered samples were cut, mounted, polished and micro etched using Keller reagent for their characterization. Mechanical testing was carried out using an Instron machine model 4468 (by triplicate). Transmission electron microscopy specimens were prepared by the focused ion beam technique.

In the plot of Fig. 1 it is noticeable a better mechanical performance (yield strength and hardness) in all samples processed by HFIS compared to the CM sample and a slight reduction in yield strength as the sintering is increased during the HFIS processing.

In the micrographs of Fig. 2 it is noticeable the obtaining of a laminar microstructure (b) after milling. Increasing the sintering time it is possible retain this microstructure (d-i). However, the grain size of samples is directly affected by the sintering time and the mechanical response was reduced. On the other hand, the porosity is drastically reduced after 3 minutes.

With CM the particular microstructure achieved by MM is almost. The grains grow at the expense of their neighbors as is shown in Fig. 2k, meanwhile, by HFIS the refined microstructure is maintained after processing Fig. 2j. 


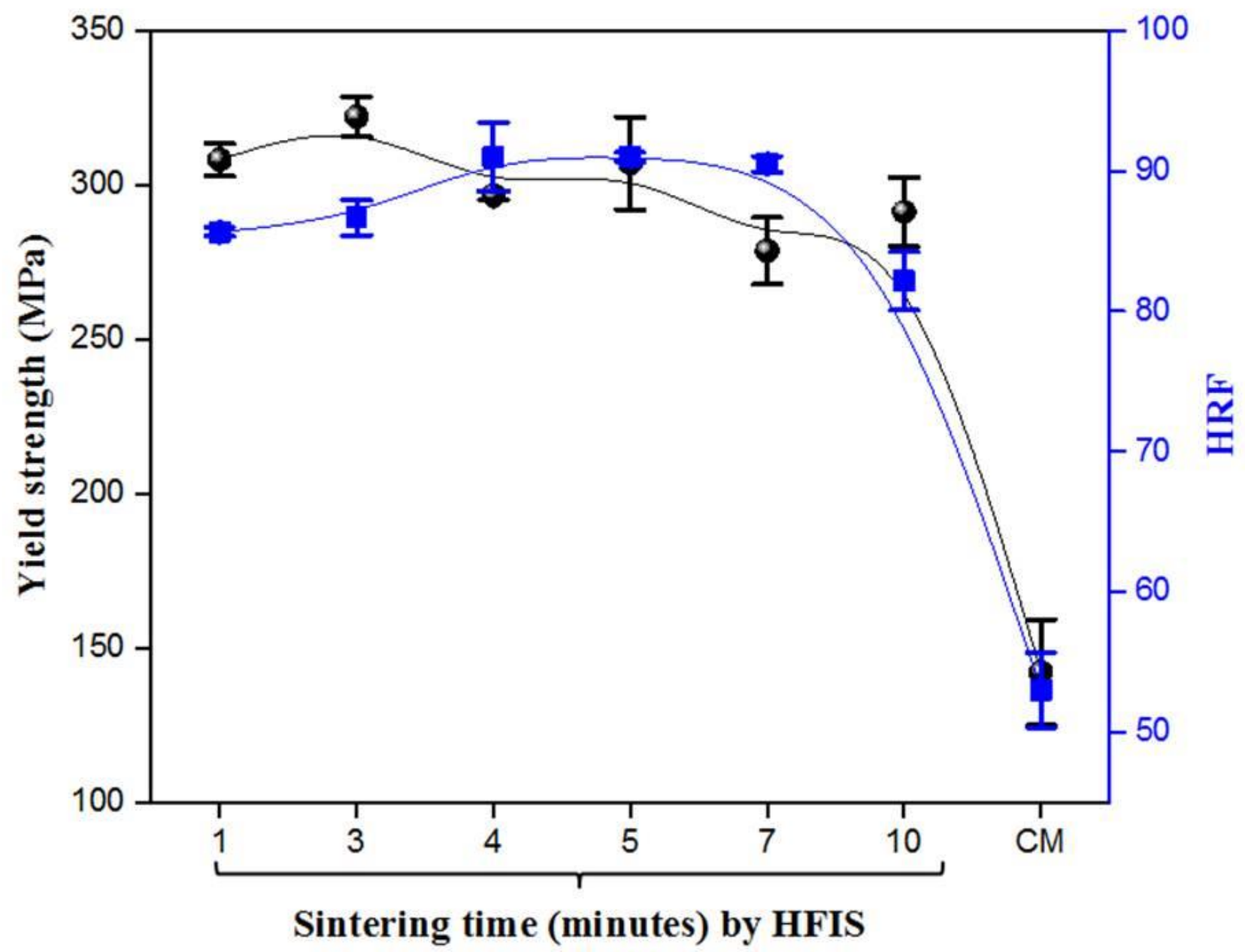

Figure 1. Figure 1.- Yield strength and Harness Rockwell F versus Sintering conditions 

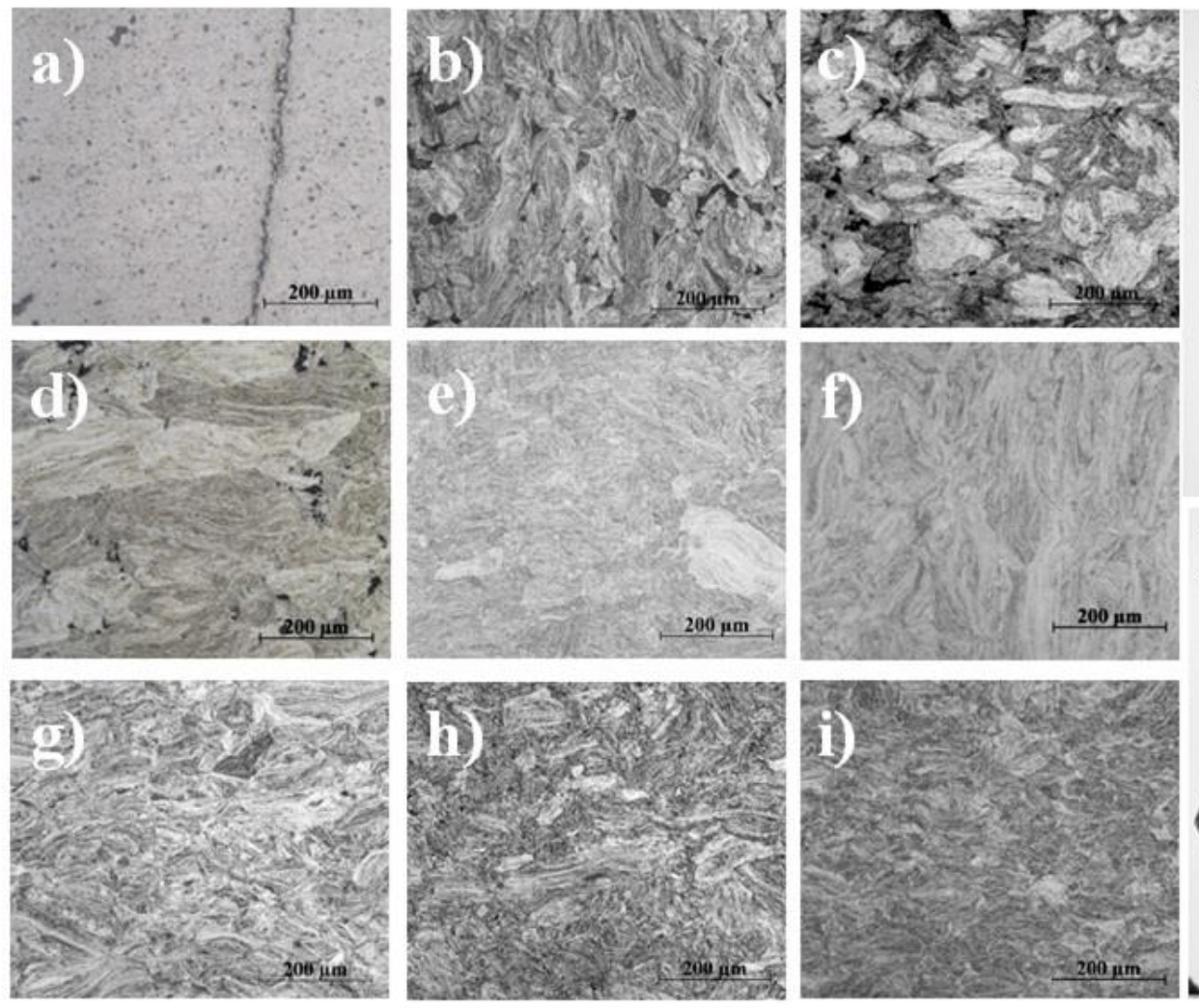
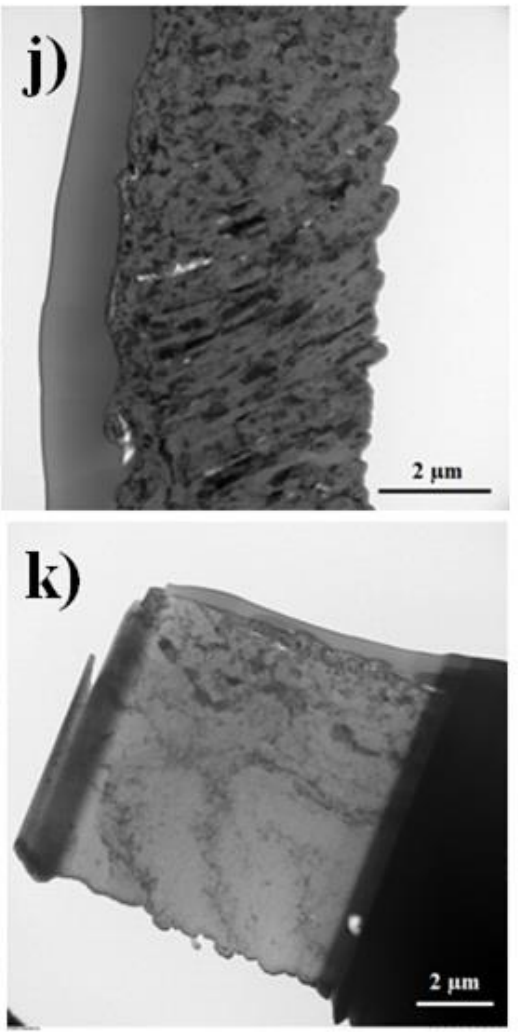

Figure 2. Figure 2.- Optical micrographs of cold compacted Al unmilled (a), milled 2h (b) and Al milled 2 hours sintered by CM (c), HFIS at 1(d), 3(e), 4(f). 5(g), 7(h) and 10 (i) minutes and TEM micrographs of HFIS sintered 3 minutes $(\mathrm{j})$ and $\mathrm{CM}$ samples $(\mathrm{k})$.

\section{References}

[1] C. Suryanarayana and I.-S. An, "Mechanical Alloying and Milling," J. Korean Powder Metall. Inst., vol. 13, no. 5, pp. 371-372, Oct. 2006, doi: 10.4150/KPMI.2006.13.5.371.

[2] V. Poojitha, T. Raghu, and V. Pandurangadu, "Microstructure evolution and mechanical behaviour of pure aluminium and aluminium alloys processed through constrained groove pressing," Mater. Today Proc., vol. 18, pp. 2335-2344, 2019, doi: 10.1016/j.matpr.2019.07.017.

[3] K.-T. L. Jong-YeolYoo, In-Jin Shon, Byung-Hyun Choi, "Fabrication and characterization of a Ni-YSZ anode support using high-frequency induction heated sintering (HFIHS)," Ceram. Int., vol. 37, pp. 569-2574, 2011. 